\title{
Performance Limits in Subband Beamforming
}

Sven Nordholm*, Ingvar Claesson and Nedelko Grbić 


\begin{abstract}
This paper analyses subband beamforming schemes mainly aimed at speech enhancement and acoustic echo suppression applications such as hands-free telephony for both mobile and office environments, internet telephony and video conferencing.

Analytical descriptions of both causal finite-length and noncausal infinite-length subband microphone array structures are given. More specifically, this paper compares finite Wiener filter performance with the noncausal Wiener solution, giving a comprehensive theoretical suppression limit.

It is shown that even short filters will yield a good approximation of the infinite solution, provided that the element spacing and temporal sampling is matched to the frequency band of interest. Typically, 10-20 FIR taps are sufficient in each subband.
\end{abstract}

Index Terms - beamforming, speech enhancement, filterbank, subband, hands-free, microphone array.

proposed SAP EDICS: 1-ENHA

corresponding address:

Sven Nordholm

Australian Telecommunications Research Institute

Curtin University of Technology

GPO BOX U 1987

Perth, WA 6845

AUSTRALIA

Email: sven@watri.uwa.edu.au

Telephone: +61-8-9380-8021

Facsimile: +61-8-9380-7254 


\section{INTRODUCTION}

The hands-free situation for telephony in cars and offices, internet telephony and video conferencing gives different enhancement requirements in order to achieve a desired speech quality. Different applications also require a different cost level on the instrumentation side. Hands-free solutions for mobile and office situations as well as internet telephony are typically low-cost products. This implies that the extra cost allowed for speech enhancement and acoustic echo cancellation is limited. Subband beamforming may be used to reduce the complexity inherent in conventional beamformers and thus better suit these low cost products.

The advantages of hands-free communication are safety, convenience and greater flexibility. A hands-free communication set-up includes at least one loudspeaker and one microphone in the same environment. This means that the microphone(s) are subject to several near-end disturbances. These near-end disturbances may result in substantial speech degradation and they are mainly caused by room reverberation and background noise. Acoustic feedback (generated at the near end) is a problem for the far-end speaker, who will hear his or her voice echoed on a 100-200 ms delay, additionally making speech intelligibility more difficult. Digital filtering may be used to obtain a similar sound quality as for hand held telephony. The filtering operations have in general strong restrictions on the induced delay.

This paper analyses a spatial diversity scheme using a subband beamforming structure which can handle these hands-free problems effectively.

Some of the existing methods of increasing the signal-to-noise ratio in hands-free mobile telephones are spectral subtraction [1], [2], [3], temporal filtering, noise cancellation, and a variety of different array techniques [4], [5], [6], [7], [8], [9], [10]. Room reverberation is most effectively handled by array techniques or proper microphone design and placement. Acoustic feedback for hands-free mobile telephony is usually addressed by conventional echo cancellation techniques [11], [12], [13], although subband methods are emerging techniques [14]. There are also possibilities to exploit the effect of masking [15], [16] in order to improve the speech quality.

In this paper novel subband beamforming structures are analyzed, which are inspired from octave band structures, closely related to wavelets.

The digital design of a beamformer consists of two coupled sampling processes, the spatial and the temporal sampling, [17]. The array input signals are bandlimited, sampled and each of the discrete-time signals are digitally filtered using a bank of filters. The same filterbank is used for 
each channel and thus all spatial properties are kept in the subbands. The output signals from the filterbank in each frequency band are temporally oversampled, hence a temporal decimation can be performed. This is the usual case for a single input signal and it is straightforward and well understood. There is a potential for using different sets of microphone elements in different subbands, corresponding to some sort of spatial decimation depending on the temporal frequency range.

This paper gives performance limits for subband beamformers for spatially spread sources, corrupted by a diffuse noise field. The gain in Signal-to-Noise plus Interference Ratio, (SNIR) is calculated for FIR filter-and-sum beamformers for different frequency bands with different sampling rates, and compares the performance with infinite Wiener filter beamforming.

The outline of the paper is as follows: In section 2, the subband beamforming scheme is presented. Signal model and both finite, and optimum infinite, Wiener solutions are described in section 3. In section 4, the performance versus the filter length is given and it is compared to the optimal noncausal infinite Wiener solutions for different sample rates. Angular resolution is also evaluated for different sensor spacing. Finally, section 5 summarizes and makes conclusions and proposes further ideas.

\section{Subband Beamformer}

Several subband adaptive beamforming schemes have been proposed [18], [19], [20], [21], [22]. There are several advantages with a subband beamformer, as compared to a conventional fullband beamformer:

- a shorter filter length in total

- better interference suppression

- a simple optional weighting in each frequency band

- faster convergence

- less computational load

The normal procedure when designing a subband beamformer is to use a scheme like the one shown in Fig. 1. One important drawback with this type of scheme is the unwanted delay caused by the filterbank. Another drawback is the problem with signal reconstruction since the signal after the interpolation becomes cyclostationary due to the aliasing between the different subbands, see [23], [24]. The recovered signal is not a stationary stochastic process even if the input is stationary. One way to circumvent these problems, to some extent, is to allow for over 
sampling in the subbands. A factor two oversampled non-uniform filterbank design is proposed in [25] where such aliasing effects are minimized together with the filterbank transformation delay. The extra parameters needed in the beamformer by using oversampling are discussed in this paper.

Another issue to address is whether the subband splitting should be performed with an FFT operation, yielding equal-bandwidth subbands or in an iterative half band filtering and decimationby-2 fashion, yielding octave subbands. The latter can also be more attractive from an auditory point of view.

\section{Analysis of the Suggested Beamforming Scheme}

Important issues to discuss and analyse are the number of FIR taps needed to achieve essentially the same performance as an optimum beamformer, and how the input signal should be divided into subbands in order to obtain an efficient structure with short FIR filters. The use of fewer filter coefficients as compared to the fullband realization will give savings in the required number of arithmetic operations, which is crucial for real time applications.

\section{A. Signal Model and Wiener Solutions}

The studied situation corresponds to an operating/adaptive phase of a subband beamformer, while analysis and experiments are performed separately for each subband.

All signal sources are assumed to be a cluster of an infinite number of point sources equally distributed in a small region in space. It should be noted that all point sources within a cluster are assumed to be independent, having the same spectral properties. This might not be the case for real spread source, such as a human mouth or a hands-free loudspeaker, where some dependency within the area/volume may be expected. However, we do not wish to resolve such temporal dependency within a cluster, rather the spatio-temporal dependency between sources and noise is in focus in this evaluation.

The response vector, in two dimensional space, from a point source location to the array is denoted $\mathbf{G}(r, \theta, \Omega)$ and it is given by

$$
\mathbf{G}(r, \theta, \Omega)=r e^{j \Omega \tau(r, \theta)}\left[\frac{1}{r_{1}} e^{-j \Omega \tau_{1}(r, \theta)}, \frac{1}{r_{2}} e^{-j \Omega \tau_{2}(r, \theta)}, \ldots, \frac{1}{r_{N}} e^{-j \Omega \tau_{N}(r, \theta)}\right]^{T}
$$

where $\Omega$ is real angular frequency and $\tau_{n}(r, \theta)$ denoting the time delay from a point source at radius $r$ and angle $\theta$ to sensor $n$, and $r_{n}$ is the distance between the source and sensor $n$. 
The response vector includes a constant, $r e^{j \Omega \tau(r, \theta)}$, which normalizes the response to unity at the origin of coordinates. In the calculation model, a spherical propagation in a free-field and homogeneous medium has been assumed. The model includes arbitrary placement of microphone elements and sources with arbitrary spectral content. The $M$ different signal sources' magnitude spectra $S_{m}(\Omega), m=1,2, \ldots, M$ with spectral densities, $S_{s_{m}}(\Omega)$, and autocorrelation functions, $r_{s_{m}}(\tau)$, are assumed to be mutually uncorrelated, i.e. the cross power spectral density, $S_{s_{l} s_{m}}(\Omega)$, is zero if $l \neq m$. All sources impinge on an array with $N$ microphone elements situated in a spherically isotropic noise field with magnitude spectrum $N_{n}(\Omega)$, i.e. the array is corrupted by an infinite number of uncorrelated noise sources along a spherical shell at a large radius.

In the analytical evaluation, the desired signal location and power spectral density is presumed to be known. The received microphone signal vector magnitude spectrum, $\mathbf{X}(\Omega)$, is given by

$$
\mathbf{X}(\Omega)=\sum_{m=1}^{M} S_{m}(\Omega) \iint_{\mathbf{A}_{m}} \mathbf{G}(r, \theta, \Omega) d \mathbf{A}+\mathbf{N}(\Omega)
$$

where the regions of integration $\mathbf{A}_{m}$ are circles/spheres in 2/3-dimensional space corresponding to each source cluster distribution, see. Fig. 2 for a case with one desired source and one jammer source with a linear array. The received array vector is arranged as,

$$
\mathbf{X}(\Omega)=\left[X_{1}(\Omega) X_{2}(\Omega) \ldots X_{N}(\Omega)\right]^{T}
$$

Without loss of analytic generality it is assumed that the desired signal is point source number one, i.e.

$$
D(\Omega)=S_{1}(\Omega) F(\Omega)
$$

where $F(\Omega)$ corresponds to an arbitrary filter. The filter, $F(\Omega)$, may be used for optional temporal weighting of the target signal.

In the sequel we assume that all input signals are sampled and correctly bandlimited with normalized angular frequency $\omega=\Omega T_{s}$, where $T_{s}$ is the sampling period. The sampled input signal vector is given by

$$
\mathbf{x}(\omega)=\left[x_{1}(\omega) x_{2}(\omega) \ldots x_{N}(\omega)\right]^{T}
$$

All the inputs are filtered through the same filterbank dividing the input signals into $Q$ bands

$$
\mathbf{x}_{q}(\omega)=H_{q}(\omega) \mathbf{x}(\omega)
$$


where $\mathbf{x}_{q}(\omega)$ is the array signal in band $q$ and $H_{q}(\omega)$ is the transfer function corresponding to the $q$ :th filter in the filterbank. These signals are decimated by a factor $P$. As will be shown later, it is beneficial to use different decimation factors in different frequency bands. The objective is to obtain efficient processing, i.e. use few parameters, while maintaining a performance close to that achieved by optimum Wiener filtering. The decimation only affects the temporal sampling. The spatial sampling, however, is determined by the geometrical interrelations between the signals on each array element. The decimated received signal vector is given by

$$
\tilde{\mathbf{x}}_{q}(\omega)=\sum_{p=0}^{P-1} \mathbf{x}_{q}\left(\frac{\omega+2 \pi p}{P}\right)
$$

and the decimated desired signal is given by

$$
\tilde{d}_{q}(\omega)=\sum_{p=0}^{P-1} d_{q}\left(\frac{\omega+2 \pi p}{P}\right)
$$

where $d_{q}(\omega)$ is the sampled desired signal in subband $q$. It should be noted that the decimation factor, $P$, is a function of $q$ as it may be chosen differently for different subbands. This dependency will be omitted for readability.

\section{B. Wiener Solution, Optimum Filter Lengths}

The subband array employs optimal/adaptive beamforming in each subband, see Fig. 1. It is therefore necessary to study the solution in each frequency band.

The power spectral density matrix for the discrete-time signals in band $q$, after decimation by factor $P$, is given by

$$
\begin{aligned}
& \mathbf{S}_{\tilde{\mathbf{x}}_{q} \tilde{\mathbf{x}}_{q}}(\omega)=\frac{1}{P} \sum_{p=0}^{P-1} \mathbf{S}_{\mathbf{x}_{q} \mathbf{x}_{q}}\left(\frac{\omega+2 \pi \cdot p}{P}\right)= \\
& \frac{1}{P} \sum_{p=0}^{P-1}\left(\sum_{m=1}^{M} S_{s_{m}}\left(\frac{\omega+2 \pi \cdot p}{P}\right)\left|H_{q}\left(\frac{\omega+2 \pi \cdot p}{P}\right)\right|^{2} \iint_{\mathbf{A}_{m}} \mathbf{G}\left(r, \theta, \frac{\omega+2 \pi \cdot p}{P T_{s}}\right) \mathbf{G}^{H}\left(r, \theta, \frac{\omega+2 \pi \cdot p}{P T_{s}}\right) d \mathbf{A}\right. \\
& \left.+\left|H_{q}\left(\frac{\omega+2 \pi \cdot p}{P}\right)\right|^{2} \mathbf{S}_{\mathbf{n n}}\left(\frac{\omega+2 \pi \cdot p}{P}\right)\right)
\end{aligned}
$$


and the cross power spectral density vector is given by

$$
\begin{aligned}
& \mathbf{S}_{\tilde{d}_{q} \tilde{x}_{q}}(\omega)=\frac{1}{P} \sum_{p=0}^{P-1} \mathbf{S}_{d_{q} \mathbf{x}_{q}}\left(\frac{\omega+2 \pi \cdot p}{P}\right)= \\
& \frac{1}{P} \sum_{p=0}^{P-1}\left(S_{s_{1}}\left(\frac{\omega+2 \pi \cdot p}{P}\right)\left|H_{q}\left(\frac{\omega+2 \pi \cdot p}{P}\right)\right|^{2} \iint_{\mathbf{A}_{1}} \mathbf{G}^{H}\left(r, \theta, \frac{\omega+2 \pi \cdot p}{P T_{s}}\right) d \mathbf{A}\right)
\end{aligned}
$$

where $T_{s}$ is the sampling period, $H_{q}(\omega)$ is the transfer function of the $q$-th subband filter and $\mathbf{G}(r, \theta, \Omega)$ denotes the spatial response vector given in Eq. (1).

The spherically isotropic noise power spectral density matrix $\mathbf{S}_{\mathbf{n n}}(\omega)$ is given by integrating infinite number of uncorrelated point sources inside a spherical shell at an infinite radius, [26]. A closed-form expression at position $(i, j)$ of the normalized noise power spectral density matrix is given by, [26],

$$
\mathbf{S}_{\mathbf{n n}}(\omega)_{(i, j)}=\frac{\sin \left(\frac{\omega \kappa_{i j}}{c T_{s}}\right)}{\frac{\omega \kappa_{i j}}{c T_{s}}}
$$

where $\kappa_{i j}$ is the distance between microphone elements of index $i$ and $j$, and $c$ is the speed of wave propagation. The noncausal Wiener solution in each subband ${ }^{1}$ minimizes the local error power spectral density in that subband with respect to the row filter vector, $\mathbf{W}_{q}(\omega)$, and can be found by expressing the orthogonality between the error, $\varepsilon_{q}[l]$, and the inputs, $\tilde{\mathbf{x}}_{q}[l]$, (this is done for the fullband case in [27])

$$
\mathbf{S}_{\varepsilon_{q} \tilde{\mathbf{x}}_{q}}(\omega)=\mathbf{S}_{\tilde{d}_{q} \tilde{\mathbf{x}}_{q}}(\omega)-\mathbf{W}_{q}(\omega) \mathbf{S}_{\tilde{\mathbf{x}}_{q} \tilde{\mathbf{x}}_{q}}(\omega)=0
$$

yielding

$$
\mathbf{W}_{q, o p t}(\omega)=\mathbf{S}_{\tilde{d}_{q} \tilde{\mathbf{x}}_{q}}(\omega) \mathbf{S}_{\tilde{\mathbf{x}}_{q} \tilde{\mathbf{x}}_{q}}^{-1}(\omega)
$$

The corresponding minimum error power spectral density is given by

$$
\mathbf{S}_{\varepsilon_{q} \varepsilon_{q}, o p t}(\omega)=\mathbf{S}_{\tilde{d}_{q} \tilde{d}_{q}}(\omega)-\mathbf{S}_{\tilde{d}_{q} \tilde{\mathbf{x}}_{q}}(\omega) \mathbf{S}_{\tilde{\mathbf{x}}_{q} \tilde{\mathbf{x}}_{q}}^{-1}(\omega) \mathbf{S}_{\tilde{\mathbf{x}}_{q} \tilde{d}_{q}}(\omega)
$$

The Wiener solution in each band expresses the optimum performance for an adaptive beamformer with infinite length noncausal filters in that frequency band.

The power spectral density matrix, $\mathbf{S}_{\tilde{\mathbf{x}}_{q} \tilde{\mathbf{x}}_{q}}(\omega)$, consists of a target signal and jamming signals corrupted with noise. We can separate $\mathbf{S}_{\tilde{\mathbf{x}}_{q} \tilde{\mathbf{x}}_{q}}(\omega)$ into two parts, one containing desired signals

${ }^{1}$ This solution does not guarantee that the fullband error is minimized since there is a leakage between the frequency bands 
and one with undesired signals

$$
\begin{aligned}
& \mathbf{S}_{\tilde{\mathbf{x}}_{q} \tilde{\mathbf{x}}_{q}}(\omega)= \\
& \frac{1}{P} \sum_{p=0}^{P-1}\left(S_{s_{1}}\left(\frac{\omega+2 \pi \cdot p}{P}\right)\left|H_{q}\left(\frac{\omega+2 \pi \cdot p}{P}\right)\right|^{2} \iint_{\mathbf{A}_{1}} \mathbf{G}\left(r, \theta, \frac{\omega+2 \pi \cdot p}{P T_{s}}\right) \mathbf{G}^{H}\left(r, \theta, \frac{\omega+2 \pi \cdot p}{P T_{s}}\right) d \mathbf{A}\right. \\
& \left.+\left|H_{q}\left(\frac{\omega+2 \pi \cdot p}{P}\right)\right|^{2} \mathbf{S}_{\nu \nu}\left(\frac{\omega+2 \pi \cdot p}{P}\right)\right) .
\end{aligned}
$$

The matrix

$$
\mathbf{S}_{\tilde{\nu}_{\mathbf{q}} \tilde{\nu}_{\mathbf{q}}}(\omega)=\frac{1}{P} \sum_{p=0}^{P-1}\left|H_{q}\left(\frac{\omega+2 \pi \cdot p}{P}\right)\right|^{2} \mathbf{S}_{\nu \nu}\left(\frac{\omega+2 \pi \cdot p}{P}\right)
$$

consists of all the undesired signals, including measurement noise, i.e.

$$
\mathbf{S}_{\nu \nu}(\omega)=\sum_{m=2}^{M} S_{s_{m}}(w) \iint_{\mathbf{A}_{m}} \mathbf{G}\left(r, \theta, \omega / T_{s}\right) \mathbf{G}^{H}\left(r, \theta, \omega / T_{s}\right) d \mathbf{A}+\mathbf{S}_{\mathbf{n n}}(\omega)+\sigma^{2} \mathbf{I}
$$

where $\sigma^{2} \mathbf{I}$ is a diagonal matrix, corresponding to uncorrelated measurement noise with variance $\sigma^{2}$. We need to apply a general form of the matrix inversion lemma to express $\mathbf{S}_{\tilde{\mathbf{x}}_{q} \tilde{\mathbf{x}}_{q}}^{-1}(\omega)$ in closed form. Assuming the overlap between different frequency bands to be zero, i.e. ideal filters $H_{q}(\omega)$ in the filterbank, the corresponding optimal performance will serve as an upper bound, independent on subband filterbank properties. Using equation (15) in equation (13) and finding an expression for $\mathbf{S}_{\tilde{d}_{q} \tilde{\mathbf{x}}}$, we get

$$
\mathbf{W}_{q, o p t}(\omega)=S_{\tilde{s}_{1}}(\omega) F(\omega) \mathbf{B}^{H} \mathbf{S}_{\tilde{\nu} \tilde{\nu}}^{-1}(\omega)\left(\mathbf{I}-\left(\mathbf{S}_{\tilde{\nu} \tilde{\nu}}^{-1}(\omega)+S_{\tilde{s}_{1}}(\omega)^{-1} F(\omega)^{-1} \mathbf{C}\right)^{-1} \mathbf{S}_{\tilde{\nu} \tilde{\nu}}^{-1}(\omega)\right)
$$

where $F(\omega)$ is the transfer function of the corresponding discrete time analogous of filter $F(\Omega)$, given in Eq. (4) and matrices $\mathbf{B}$ and $\mathbf{C}$ are given by

$$
\begin{aligned}
& \mathbf{B}=S_{\tilde{s}_{1}}(\omega) \iint_{\mathbf{A}_{1}} \mathbf{G}^{H}\left(r, \theta, \omega / T_{s}\right) d \mathbf{A} \\
& \mathbf{C}=S_{\tilde{s}_{1}}(\omega) \iint_{\mathbf{A}_{1}} \mathbf{G}\left(r, \theta, \omega / T_{s}\right) \mathbf{G}^{H}\left(r, \theta, \omega / T_{s}\right) d \mathbf{A} .
\end{aligned}
$$

The beamformer gain in signal-to-noise plus interference ratio (SNIR) is given by

$$
\operatorname{GAIN}_{S N I R}=\frac{\mathbf{W}_{q, \text { opt }}^{H}(\omega) \mathbf{S}_{\mathbf{s}_{1} \mathbf{s}_{1}}(\omega) \mathbf{W}_{q, \text { opt }}(\omega)}{\mathbf{W}_{q, \text { opt }}^{H}(\omega) \mathbf{S}_{\nu \nu}(\omega) \mathbf{W}_{q, \text { opt }}(\omega)}
$$

where $\mathbf{S}_{\mathbf{s}_{1} \mathbf{s}_{1}}(\omega)$ is the received power spectral density matrix of the desired source and $\mathbf{S}_{\nu \nu}(\omega)$ is given in (16). The corresponding expression for the error power spectral density is given by

$$
S_{\varepsilon_{q} \varepsilon_{q}, o p t}(\omega)=1-|F(\omega)|^{2} \mathbf{B}^{H} \mathbf{S}_{\tilde{\nu} \tilde{\nu}}^{-1}(\omega)\left(\mathbf{I}-\left(\mathbf{S}_{\tilde{\nu} \tilde{\nu}}^{-1}(\omega)+S_{\tilde{s}_{1}}(\omega)^{-1} F(\omega)^{-1} \mathbf{C}\right)^{-1} \mathbf{S}_{\tilde{\nu} \tilde{\nu}}^{-1}(\omega)\right) \mathbf{B} .
$$


As a special case, when each source is modeled as a point source, i.e. assuming each corresponding source signal power spectral density matrix being a rank one matrix, the simplest form of the matrix inversion lemma is applicable and a compact formula for the solution is given by

$$
\mathbf{W}_{q, o p t}(\omega)=\frac{S_{\tilde{s}_{1}}(\omega) F(\omega) \mathbf{G}_{s_{1}}^{H}\left(r, \theta, \omega / T_{s}\right) \mathbf{S}_{\tilde{\nu} \tilde{\nu}}^{-1}(\omega)}{S_{\tilde{s}_{1}}(\omega) \mathbf{G}_{s_{1}}^{H}\left(r, \theta, \omega / T_{s}\right) \mathbf{S}_{\tilde{\nu} \tilde{\nu}}^{-1}(\omega) \mathbf{G}_{s_{1}}\left(r, \theta, \omega / T_{s}\right)+1} .
$$

The corresponding expression for the error power spectral density is given by

$$
S_{\varepsilon_{q} \varepsilon_{q}, o p t}(\omega)=\frac{S_{\tilde{s}_{1}}(\omega)|F(\omega)|^{2}}{S_{\tilde{s}_{1}}(\omega) \mathbf{G}_{s_{1}}^{H}\left(r, \theta, \omega / T_{s}\right) \mathbf{S}_{\tilde{\nu} \tilde{\nu}}^{-1}(\omega) \mathbf{G}_{s_{1}}\left(r, \theta, \omega / T_{s}\right)+1} .
$$

Once the optimum filters, $\mathbf{W}_{q, \text { opt }}(\omega)$, in each band are found, the best performance for a linear system in that frequency band is obtained. This optimal solution can then be a benchmark for the FIR solutions. Note, in a theoretical case, i.e. a situation with perfect non-overlapping filters, the performance in each subband is the same as for a fullband beamformer, viewed over the same frequency region.

\section{Wiener Solution, FIR filters}

The FIR solution approximates the optimum Wiener filter with better accuracy as the filter length increases. It is however not a simple relationship, since there are two more variable parameters, the frequency band and the decimation factor.

It is straightforward to formulate the optimal FIR solution in each frequency band. The task for the FIR filters is to minimize the output error, $\varepsilon_{q}[l]$, in subband $q$.

The optimal FIR filter is found by minimizing

$$
E\left(\left|\varepsilon_{q}[l]\right|^{2}\right)=\mathbf{r}_{\tilde{d}_{q} \tilde{d}_{q}}[0]-\mathbf{R}_{\tilde{d}_{q} \tilde{\mathbf{x}}_{q}} \mathbf{w}_{q}-\mathbf{w}_{q}^{H} \mathbf{R}_{\tilde{\mathbf{x}}_{q} \tilde{d}_{q}}+\mathbf{w}_{q}^{H} \mathbf{R}_{\tilde{\mathbf{x}}_{q} \tilde{\mathbf{x}}_{q}} \mathbf{w}_{q}
$$

where $\mathbf{w}_{q}$ is a stacked FIR tap vector where the filter weights from each FIR filter of length $L$ are stacked on top of each other, creating a vector of length $L N$,

$$
\mathbf{w}_{q}^{T}=\left[\begin{array}{llll}
\mathbf{w}_{1}^{T} & \mathbf{w}_{2}^{T} & \ldots & \mathbf{w}_{N}^{T}
\end{array}\right]
$$

where

$$
\mathbf{w}_{n}^{T}=\left[\begin{array}{lllll}
w_{n}(0) & w_{n}(1) & \ldots & w_{n}(L-1)
\end{array}\right] \quad n=1,2, \cdots, N .
$$

The correlation matrix $\mathbf{R}_{\tilde{\mathbf{x}}_{q} \tilde{\mathbf{x}}_{q}}$ can be expressed using the temporal and spatial response vectors, $\mathbf{E}(\Omega)$ and $\mathbf{G}(r, \theta, \Omega)$, respectively as 


$$
\begin{aligned}
& \mathbf{R}_{\tilde{\mathbf{x}}_{q} \tilde{\mathbf{x}}_{q}}=\frac{1}{P} \int_{\omega_{q}}^{P-1} \sum_{p=0} \mathbf{R}_{\mathbf{x}_{q} \mathbf{x}_{q}}\left(\frac{\omega+2 \pi \cdot p}{P}\right) d \omega= \\
& \frac{1}{P} \int_{\omega_{q}}^{P-1} \sum_{p=0}^{M}\left(\sum_{m=1}^{M} S_{s_{m}}\left(\frac{\omega+2 \pi \cdot p}{P}\right)\left|H_{q}\left(\frac{\omega+2 \pi \cdot p}{P}\right)\right|^{2} \cdot\right. \\
& \iint_{\mathbf{A}_{m}} \mathbf{E}\left(\frac{\omega+2 \pi \cdot p}{P T_{s}}\right) \mathbf{E}^{H}\left(\frac{\omega+2 \pi \cdot p}{P T_{s}}\right) \otimes \mathbf{G}\left(r, \theta, \frac{\omega+2 \pi \cdot p}{P T_{s}}\right) \mathbf{G}^{H}\left(r, \theta, \frac{\omega+2 \pi \cdot p}{P T_{s}}\right) d \mathbf{A}+ \\
& \left.\left|H_{q}\left(\frac{\omega+2 \pi \cdot p}{P}\right)\right|^{2} \mathbf{R}_{\mathbf{n n}}\left(\frac{\omega+2 \pi \cdot p}{P}\right)\right) d \omega+\sigma^{2} \mathbf{I},
\end{aligned}
$$

where $\omega_{q}$ denotes the angular frequency region contained in subband number $q$ and the symbol $\otimes$ denotes the Kronecker matrix multiplication. The temporal response vector is given by

$$
\mathbf{E}(\Omega)=e^{j \frac{L}{2} \Omega T_{s}}\left[1, e^{-j \Omega T_{s}}, \ldots, e^{-j(L-1) \Omega T_{s}}\right]^{T}
$$

which is normalized to the center lag of the FIR filters by the constant $e^{j \frac{L}{2} \Omega T}$. The spatiotemporal noise correlation matrix is given by, [26],

$$
\mathbf{R}_{\mathbf{n n}(i \cdot k, j \cdot l)}(\omega)=\cos (\omega(k-l)) \frac{\sin \left(\frac{\omega \kappa_{i j}}{c T_{s}}\right)}{\frac{\omega \kappa_{i j}}{c T_{s}}}
$$

at position $(i \cdot k),(j \cdot l)$ where $i$ and $j$ denotes sensor index positions, and $k$ and $l$ denotes time lag positions, for row and column, respectively. Parameter $T_{s}$ is the sampling period and $\kappa_{i j}$ is the distance between sensors $i$ and $j$.

The cross correlation vector is expressed straightforwardly

$$
\begin{aligned}
& \mathbf{R}_{\tilde{d}_{q} \tilde{\mathbf{x}}_{q}}=\frac{1}{P} \int_{\omega_{q}} \sum_{p=0}^{P-1} \mathbf{R}_{d_{q} \mathbf{x}_{q}}\left(\frac{\omega+2 \pi \cdot p}{P}\right)= \\
& \frac{1}{P} \int_{\omega_{q}} \sum_{p=0}^{P-1}\left(S_{s_{1}}\left(\frac{\omega+2 \pi \cdot p}{P}\right)\left|H_{q}\left(\frac{\omega+2 \pi \cdot p}{P}\right)\right|^{2} \cdot \iint_{\mathbf{A}_{1}} \mathbf{E}^{H}\left(\frac{\omega+2 \pi \cdot p}{P T_{s}}\right) \otimes \mathbf{G}^{H}\left(r, \theta, \frac{\omega+2 \pi \cdot p}{P T_{s}}\right) d \mathbf{A}\right) d \omega .
\end{aligned}
$$

The optimum weights are given by

$$
\mathbf{w}_{q, o p t}=\mathbf{R}_{\tilde{d}_{q} \tilde{\mathbf{x}}_{q}} \mathbf{R}_{\tilde{\mathbf{x}}_{q} \tilde{\mathbf{x}}_{q}}^{-1}
$$

and the minimum output power in frequency band $q$ is given by

$$
E\left(\left|\varepsilon_{q}[l]\right|^{2}\right)=\mathbf{r}_{\tilde{d}_{q} \tilde{d}_{q}}[0]-\mathbf{R}_{\tilde{d}_{q} \tilde{\mathbf{x}}_{q}} \mathbf{R}_{\tilde{\mathbf{x}}_{q} \tilde{\mathbf{x}}_{q}}^{-1} \mathbf{R}_{\tilde{\mathbf{x}}_{q} \tilde{d}_{q}}
$$


The optimization is performed separately in each frequency band. Theoretical calculations indicate that in order to obtain a certain gain in SNIR, less total weights are needed when minimizing equation (31) for each subband, in comparison to the fullband case. This is however outside the scope of this paper.

\section{Analysis and Calculations}

In this section we present fundamental calculations based on a linear array. Extension to the general case, with arbitrary microphone placement, is straightforward. The results serve as an aid to decide appropriate filter lengths and decimation factors for each subband and establish angular resolution with different sensor spacing. The evaluation is separated into different frequency bands, low frequencies $250-500 \mathrm{~Hz}$, mid-low frequencies 500-1000, mid-high frequencies 1000$2000 \mathrm{~Hz}$ and high frequencies 2000-4000 Hz. The idea has been to keep the relative bandwidth constant. Results with varying element spacing, and different angles of interference, are included together with endfire performance. The task in this paper is to develop theoretical limits and to facilitate design ideas for subband beamforming. In all calculations it is assumed that one desired source and one interfering source, corresponding to a hands-free loudspeaker, are present. Further, the signal of interest and the interfering source are assumed to have equal power. The microphone array is a linear array with five equally spaced elements. The diffuse noise SNR is set to $30 \mathrm{~dB}$, i.e the power of the uncorrelated noise sources inside a theoretical spherical shell is $30 \mathrm{~dB}$ below the source power. The independent measurement noise variance $\sigma^{2}$, given in Eqs. (16) and (26), is set at an signal-to-independent noise ratio of $50 \mathrm{~dB}$. The angle between the jammer source and array axis, is denoted by $\theta$ and the angle between the desired source and the array axis is denoted by $\phi$, see Fig. 2 .

\section{A. Finite Filter Lengths, Low-Frequency band 250-500 Hz}

The array element spacing is chosen such that it matches half the wavelength for the upper frequency in the telephone bandwidth $(300-3400 \mathrm{~Hz})$, namely $5 \mathrm{~cm}$. The angle of the desired source $\phi$ is 90 degrees and the angle of jammer source, $\theta$, is 60 degrees. Performance curves of gain in Signal-to-Noise plus Interference Ratio $\left(\operatorname{GAIN}_{S N I R}\right)$, for the low-frequency region 250$500 \mathrm{~Hz}$, are presented in the upper part of Fig. 3. The optimal Wiener filter performance is plotted as the solid line. The sampling frequency is chosen as 1,2,4,8 and $16 \mathrm{kHz}$. It can be seen that a sampling rate of $1 \mathrm{kHz}$ gives best performance with FIR filter length of fewer than 10 
taps. Each doubling of sample frequency leads to an increase of 5-10 FIR filter taps in order to reach the optimal Wiener filter performance. The differences among the different sampling rates are small, approximately $1 \mathrm{~dB}$ at FIR lengths above 10. From a computational point of view it is beneficial to use as low a sampling rate as possible. However, by allowing oversampling, fewer restrictions are imposed on the filterbank and aliasing effects may be reduced [25]. By using an oversampling of factor two (as is done in [25]), we get an increase of needed FIR parameters by 5 taps, which is a moderate extra computational cost. The maximum GAIN $_{S N I R}$ is approximately $12 \mathrm{~dB}$.

\section{B. Finite Filter Lengths, Mid-Low Frequency Band 500-1000 Hz}

In the frequency band $500-1000 \mathrm{~Hz}$, the relation between the FIR filter lengths and the GAIN $_{S N I R}$ performance is given in the bottom of Fig. 3. A similar relation to that of the lower frequency band can be seen although the needed FIR filter length is slightly larger. With approximately 12 FIR taps we get near optimal performance with the critical sampling of $2 \mathrm{kHz}$. The maximum performance is approximately $14 \mathrm{~dB}$.

\section{Finite Filter Lengths, Mid-High Frequency Band 1000-2000 Hz}

The relation of SNIR versus FIR filter length for the mid-frequency band, 1000-2000 kHz is given in the upper plot of Fig. 4. This range exhibits a slightly reduced dependency on the sampling rate. The Nyquist sampling rate, $4 \mathrm{kHz}$, gives the best result and the required number of filter taps is the same as for the low-frequency range, approximately 15 taps. The maximum GAIN $_{S N I R}$ is slightly above $15 \mathrm{~dB}$ and it is increased when compared to the low-frequency region.

\section{Finite Filter Lengths, High-Frequency Band 2000-4000 Hz}

In the high-frequency region a few more FIR filter taps are needed in order to achieve the optimum Wiener filter limit as can be seen in the lower plot of Fig. 4. Approximately 18 taps are needed with $8 \mathrm{kHz}$ sample rate and the maximum GAIN $_{S N I R}$ is $17 \mathrm{~dB}$.

\section{E. Angular Dependence}

In this section the angular dependency of the jammer is studied for two different frequency bands with different sensor spacing. Results are presented for two cases; one with pure isotropic background noise and one with pure independent noise, i.e. the noise power spectral density matrix given in Eq. (16) consists of either $\mathbf{S}_{\mathbf{n n}}(\omega)$ with $\sigma^{2}=0$ and in the other case it consists 
of $\mathbf{S}_{\mathbf{n n}}(\omega)=\sigma^{2} \cdot \mathbf{I}$ with $\sigma^{2}$ equal to $30 \mathrm{~dB}$ below the source power. The frequency regions studied are $250-500 \mathrm{~Hz}$, presented in upper plot of Fig. 5 and the frequency range $500-1000 \mathrm{~Hz}$, given in lower plot of Fig. 5, for the case with pure isotropic noise. The same plots are given in Fig. 6 for the case with independent noise alone. Sensor spacing is chosen as 0.05, 0.15 and $0.30 \mathrm{~m}$ and the FIR filter length is 15 for all cases. Critical sampling is used for the scenarios, i.e. the sampling rate is chosen as twice the highest frequency.

It can be seen from these figures that a maximum GAIN $_{S N I R}$ is reached for jammer angle at about 120 degrees for the diffuse noise field. Performance drops monotonically above this angle. Performance variations with different sensor sampling distances are small in the low frequency band while it is quite large for the higher frequency band. For the independent noise case, all curves are strictly monotonic except for the curve in frequency region 500-1000 Hz, with sensor spacing of $0.3 \mathrm{~m}$. This latter is an effect of grating lobes, caused by spatial aliasing effects [26].

\section{F. Source Endfire Performance}

Performance of the optimal Wiener filter when the desired source angle is varied is given in Fig. 7 for the different frequency bands. The source is varied between 90 and 180 degrees. The jammer source angle is set at 30 degrees separation from the source angle. The upper plot shows the case with diffuse noise field, while the lower plot shows the case of pure independent noise. The results show that performance drops in a region around 110-150 degrees for the case with diffuse noise field. This is related to the similarities of the noise power spectral density matrix and the source power spectral density matrix for these angles of arrivals, [26]. The performance drops naturally to minimum for all cases at the endfire angle, where the spatial point of symmetri appears. It should be noted that endfire performance does have a strong dependence on the simulation setup.

\section{G. Frequency-Spatial Response}

The magnitude responses for two cases with optimal FIR subband beamforming filters, with the number of taps equal 15, is shown in Fig. 8 and 9, in frequency regions 250-500 Hz and 500$1000 \mathrm{~Hz}$, respectively. The responses are plotted versus frequency and angle, while the radius is kept constant at $1 \mathrm{~m}$, according to Fig. 2. The angle of the desired source, $\phi$, is 60 degrees and the angle of jammer source $\theta$ is 120 degrees. It can be seen clearly that a region of angles are simultaneously suppressed around the center angle of the jammer. The angular spread of the 
jammer is approximately 6 degrees for the evaluated scenario (see Fig 2.)

\section{Conclusions and Future Work}

The results show that through subband processing it is possible to keep the total number of adaptive parameters at a low level and still achieve near-optimal performance relative to the infinite length Wiener solution. Conventional FFT-splitting into uniform bandwidth subbands is known to be beneficial when it comes to implementation issues, but it will, however, require non-uniformly distributed adaptive FIR filter lengths, across the subbands. With octave band procedures, and by matching the temporal sampling to each subband, it is shown that the number of parameters is nearly constant across the subbands, and thus the spatial and temporal properties are exploited in an efficient manner. We believe that this structure should be considered in broadband high-quality conference telephony where complexity issues are important as well. Typically 10-20 tap long FIR filters are needed per microphone in each subband with critical sampling in each subband. By using an oversampling of factor two, the needed number of FIR filter taps increases to approximately 15-25.

Filterbank properties and their relations to subband beamforming performance are important to analyse. This, together with filterbank design for beamforming applications will be included in future work.

\section{REFERENCES}

[1] J. R. Deller jr., J. G. Proakis and J. H. L. Hansen, Discrete-Time Processing of Speech Signals, Macmillan, 1993.

[2] S. F. Boll, "Suppression of Acoustic Noise in Speech Using Spectral Subtraction", IEEE Transactions on Acoustics, Speech, and Signal Processing, Vol. ASSP-27, no. 2, pp. 113-120, April 1979.

[3] J. Yang, "Frequency Domain Noise Suppression Approaches in Mobile Telephone Systems", Proceedings of ICASSP-93, Vol. II, pp. 363-366, April 1993.

[4] Y. Kaneda and J. Ohga "Adaptive Microphone-Array System for Noise Reduction", IEEE Transactions on Acoustics, Speech, and Signal Processing, Vol. ASSP-34, no. 6, pp. 1391-1400, December 1986.

[5] S. Nordholm, I. Claesson and B. Bengtsson "Adaptive Array Noise Suppression of Handsfree Speaker Input in Cars", IEEE Transactions on Vehicular Technology, Vol. 42, no. 4, pp. 514-518, November 1993.

[6] Y. Grenier and M. Xu "An Adaptive Array for Speech Input in Cars", in Proceedings of International Symposium on Automotive Technology and Automation (ISATA), 1990.

[7] S. Nordholm, I. Claesson, S. Nordebo and M. Dahl, "Hands-Free Mobile Telephony by Means of an Adaptive Microphone Array", in Proceedings of the 128th Meeting of the Acoustical Society of America, invited presentation, Austin TX, in JASA, Vol. 96, no. 5, pt. 2, p. 3244, November 1994. 
[8] M. Dahl and I. Claesson, "Acoustic noise and echo cancelling with microphone array", IEEE transactions on Vehicular Technology, Vol. 48, Issue: 5, pp. 1518-1526 Sept. 1999.

[9] S. Nordholm, I. Claesson and M. Dahl, "Adaptive Microphone Array Employing Calibration Signals: An Analytical Evaluation", IEEE transactions on Speech and Audio processing, Vol. 7, Issue: 3, pp. 241-252, May 1999.

[10] I. Claesson and S. Nordholm, "A Spatial Filtering Approach to Robust Adaptive Beamforming", IEEE Transactions on Antennas and Propagation, Vol. 40, no. 9, pp. 1093-1096, September 1992.

[11] M.M. Sondhi and D.A. Berkley, "Silencing Echoes in the Telephone Network" Proc. IEEE, Vol 68, pp. $948-963$.

[12] D.G. Messerschmidt, "Echo Cancellation in Speech and Data Transmission" IEEE J. Sel. Areas Commun., Vol SAC-2, pp. 283-297.

[13] M.M. Sondhi and W. Kellermann "Adaptive Echo Cancellation for speech signal", Chapter 11 in Advances in Speech Signal Processing, Edited by S. Furui and M. Sondhi, Dekker, New York, 1992.

[14] P. A. Naylor, O. Tanrikulu and A. G. Constantinidis, "Subband Adaptive Filtering for Acoustic Echo Control using Allpass Polyphase IIR filter banks", IEEE Trans. on Speech and Audio Processing, Vol. 6, no. 2, Mar. 1998.

[15] Y. Ephraim and H. L. van Trees, "A Signal Subspace approach for Speech Enhancement", IEEE Transactions on Speech and Audio Processing, Vol. 3, no. 4, pp. 251-266, July 1995.

[16] N. Virag, "Speech Enhancement Based on Masking Properties of the Auditory System", Proceedings of ICASSP-95, Vol. I, pp. 796-799, 1995.

[17] S. Nordebo, "Robust Broadband Beamforming and Digital Filter Design", Doctoral thesis, 1995:173 D, ISSN 0348-8373, Luleå University of Technology, September 1995.

[18] J.M. Khalab and M.K. Ibrahim, "Enhanced beamforming techniques for broad-band satellite applications", in 3rd European Conference on Satellite Communications - ECSC-3, p. xv+429, 55-9, Nov. 1993.

[19] I.I. Jouny and M.G. Amin, "Performance of uniform and nonuniform subband constrained adaptive beamforming under broadband correlated arrivals", in Proceedings of SPIE - Int. Soc. Opt. Eng. (USA), Vol. 2756, p. 238-48, April 1996.

[20] F. Lorenzelli, A. Wan and K. Yao, "Broadband array processing using subband techniques", in Proceedings of ICASSP-96, Vol. V, pp. 2876-2879, May 1996.

[21] N. Grbić and S. Nordholm, "Soft constrained subband beamforming for hands-free speech enhancement," in IEEE International Conference on Acoustics, Speech and Signal Processing, May 2002, vol. 1, pp. 885-888.

[22] S. Affes and Y. Grenier, "A signal subspace tracking algorithm for microphone array processing of speech", IEEE trans. of Speech and Audio Processing, Vol. 5, No. 5, pp. 425-437, Sept, 1997.

[23] V. P. Sathe and P. P. Vaidyanathan, "Effects of Multirate Systems on the Statistical Properties of Random Signals", IEEE Transactions on Signal Processing, Vol. ASSP-41, no. 1, pp. 131-146, January 1993.

[24] A. Gilliore and M. Vetterli, "Adaptive Filtering in Subbands with Critical Sampling: Analysis, Experiments, and Applications to Acoustic Echo Cancellation", IEEE Transactions on Signal Processing, Vol. ASSP-48, no. 8, pp. 1862-1875, August 1992.

[25] J. M. de Haan, N. Grbić, I. Claesson and S. Nordholm, "Design and Evaluation of Nonuniform DFT Filter 


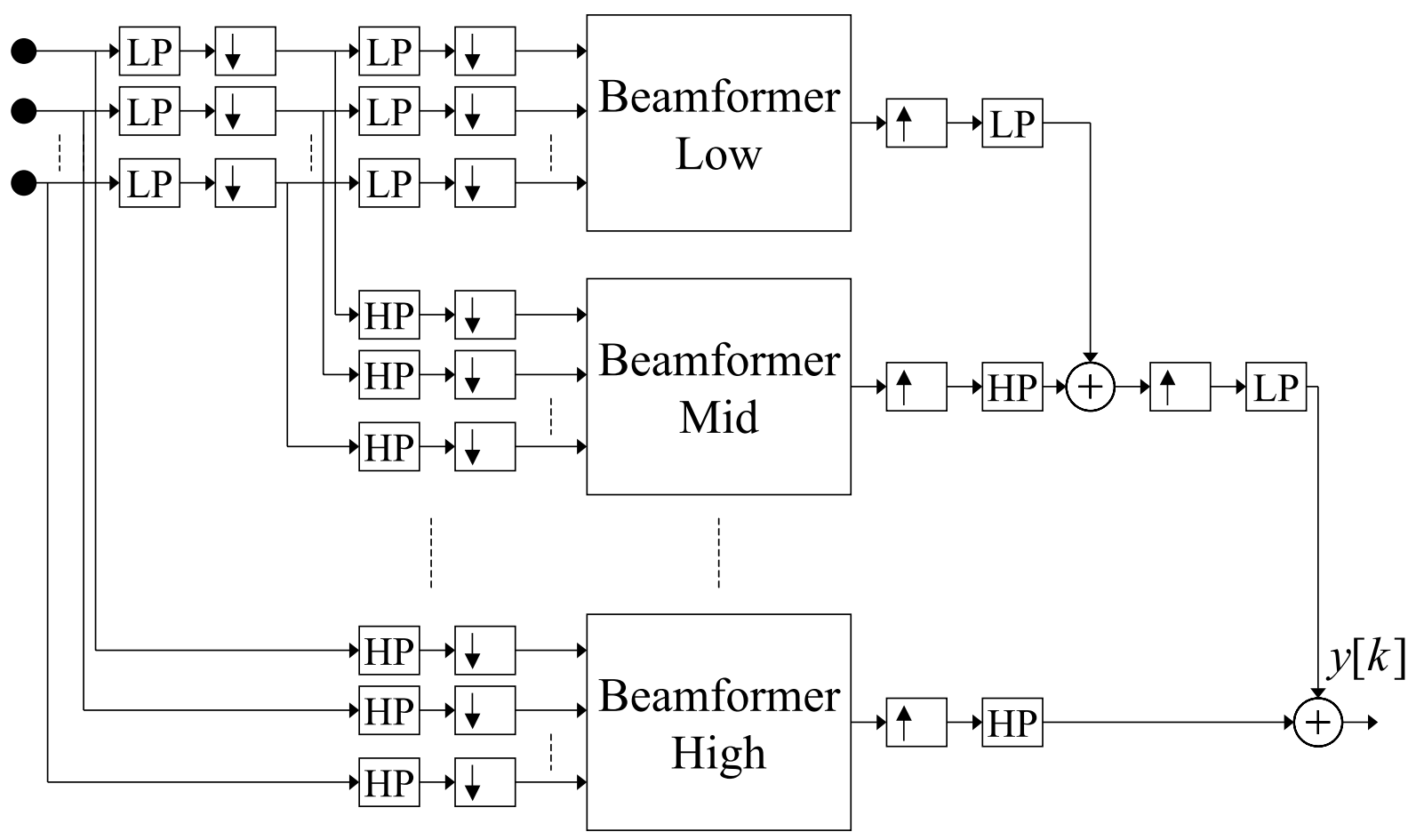

Fig. 1. Subband beamforming scheme.

banks in Subband Microphone Arrays," in IEEE International Conference on Acoustics, Speech and Signal Processing, May 2002, vol. 2, pp. 1173-1176.

[26] D. Johnson and D. Dudgeon, "Array Signal Processing - Concepts and Techniques", Prentice Hall, ISBN 0-13-048513-6, 1993.

[27] B. Widrow and S. D. Stearns, "Adaptive Signal Processing", Prentice Hall, Englewood Cliffs, NJ, 1985. 


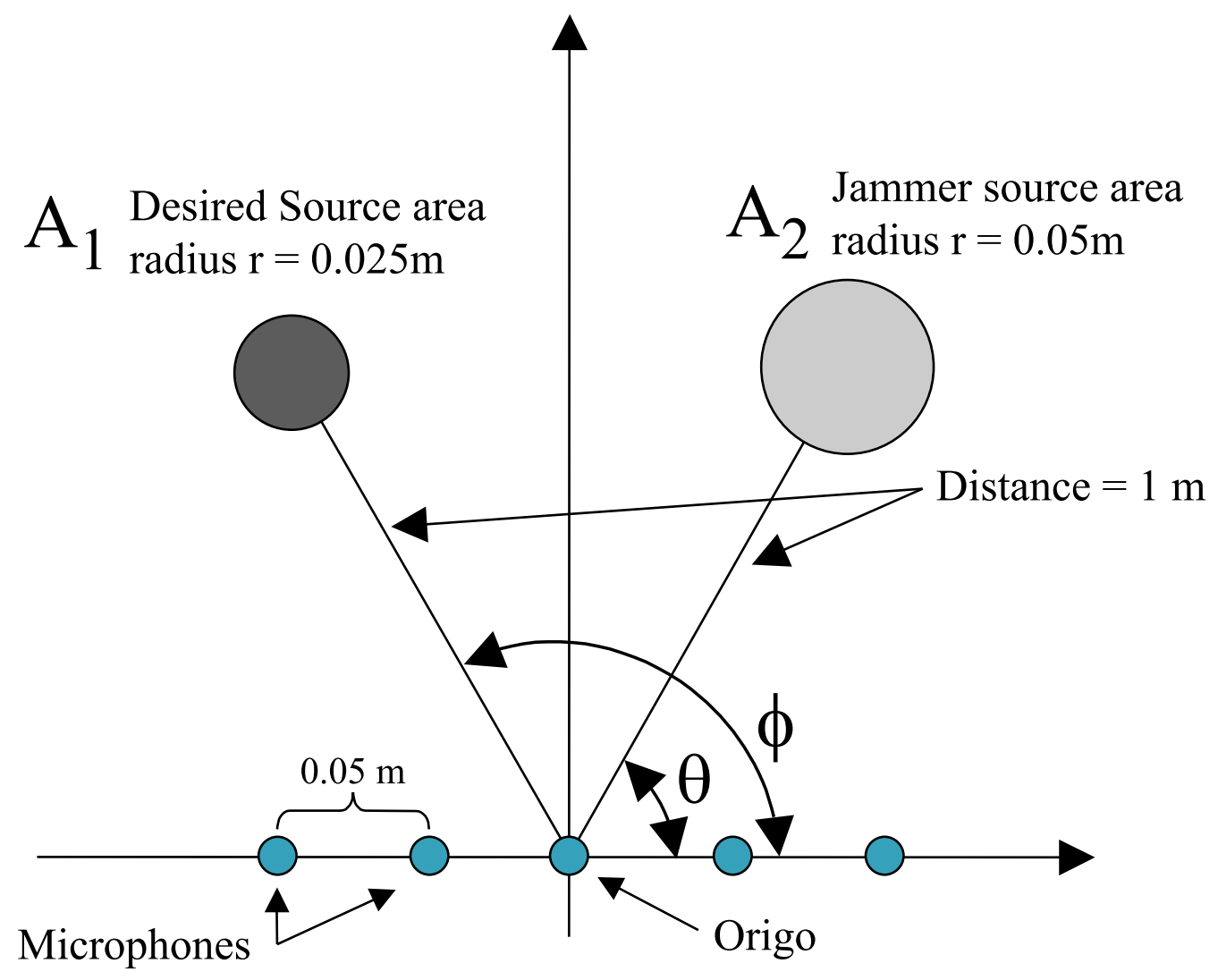

Fig. 2. Microphone array and source signal geometry. 


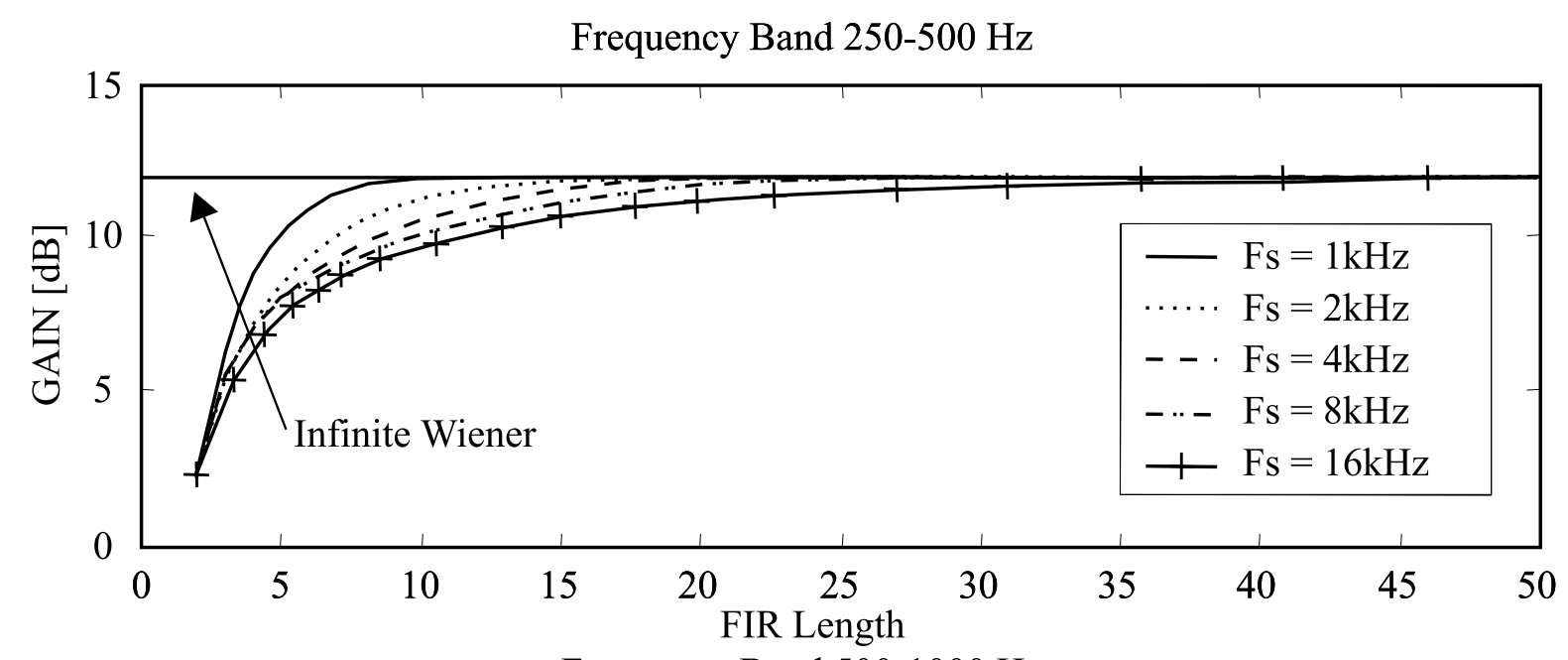

Frequency Band 500-1000 Hz

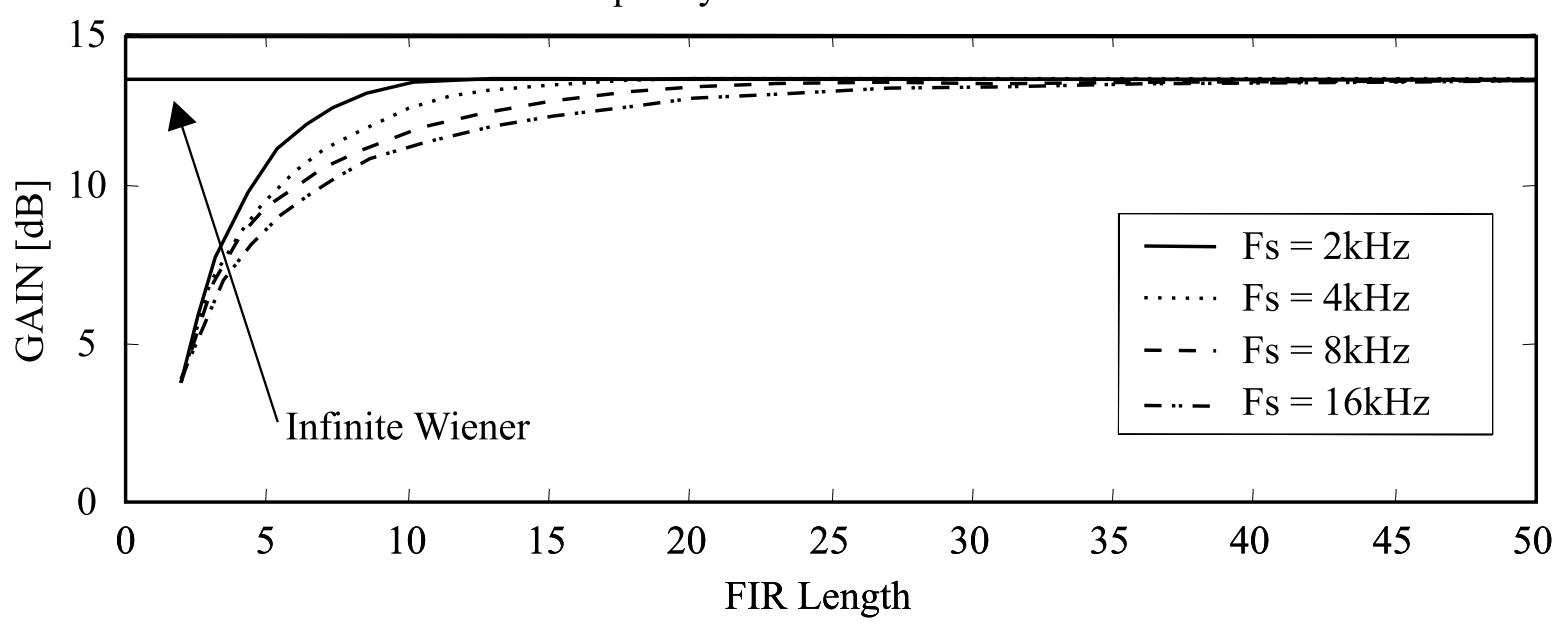

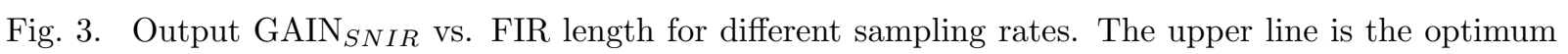
noncausal Wiener Filter limit. Linear array with $\mathrm{N}=5$, sensor distance is $0.05 \mathrm{~m}, \mathrm{SNR}=30 \mathrm{~dB}$, $\mathrm{SIR}=0 \mathrm{~dB}, \theta=60$ degrees, $\phi=90$ degrees. Upper figure - frequency band 250-500 Hz, Lower figure - Frequency band 500-1000 Hz. 

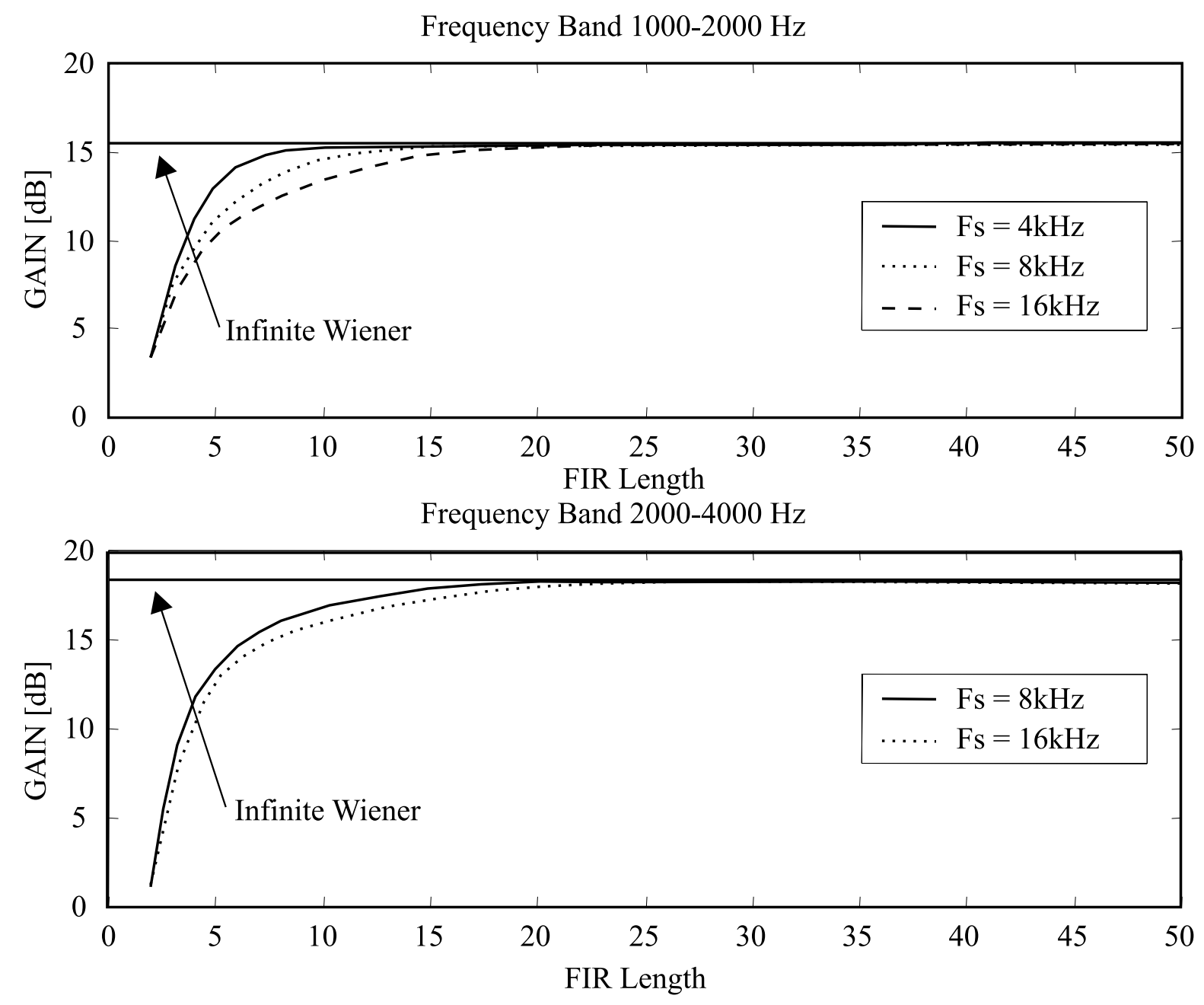

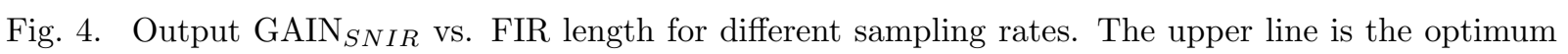
noncausal Wiener Filter limit. Linear array with $\mathrm{N}=5$, sensor distance is $0.05 \mathrm{~m}, \mathrm{SNR}=30 \mathrm{~dB}$, $\mathrm{SIR}=0 \mathrm{~dB}, \theta=60$ degrees, $\phi=90$ degrees. Upper figure - frequency band 1000-2000 Hz, Lower figure - Frequency band 2000-4000 Hz. 
Frequency Band 250-500 Hz

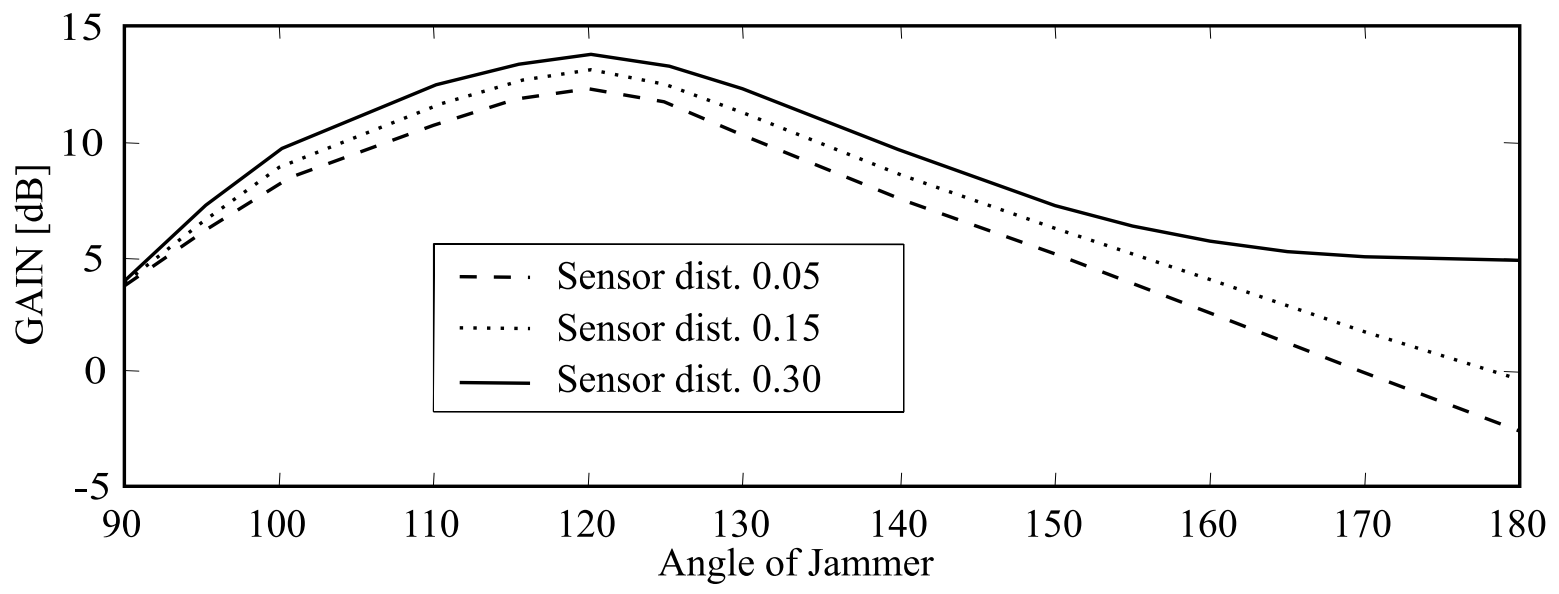

Frequency Band 500-1000 Hz

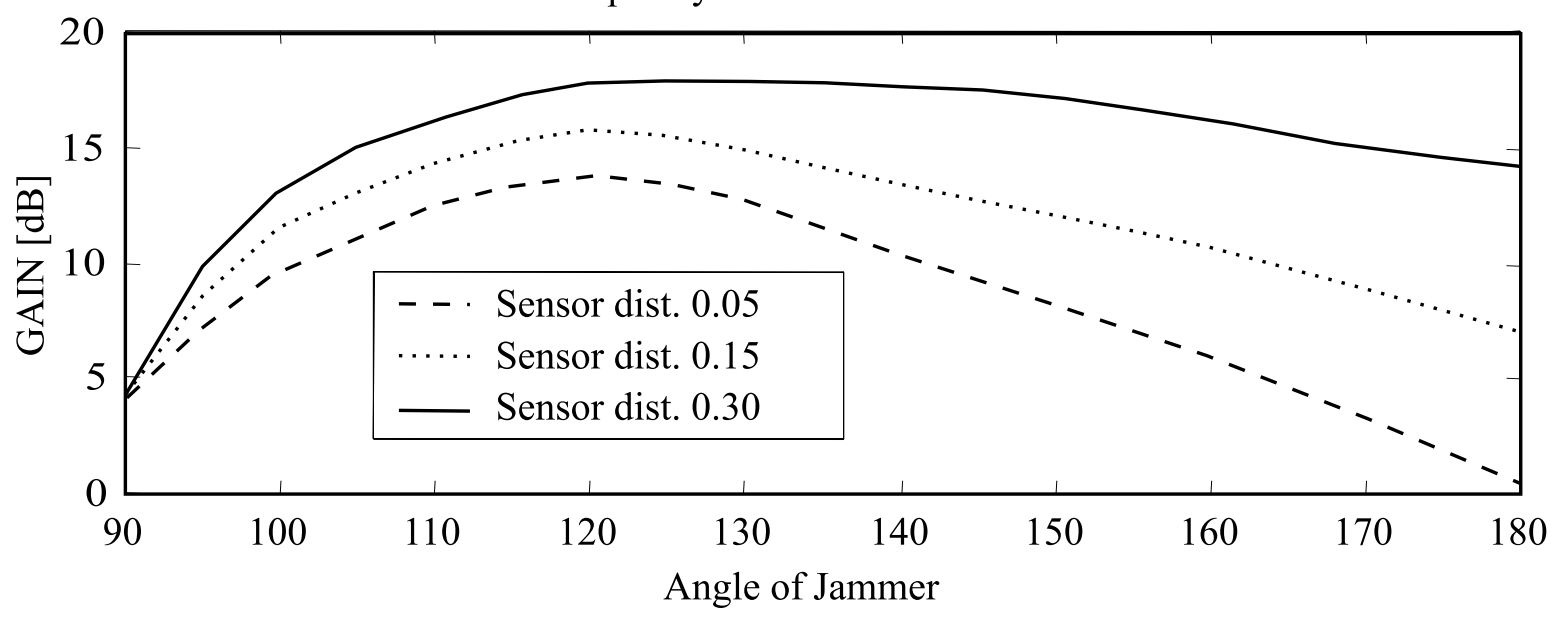

Fig. 5. Output GAIN $_{S N I R}$ vs. angle of arrival of the jammer in diffuse noise field. Linear array with $\mathrm{N}=5$, sensor distance is chosen as $0.05,0.15$ and $0.30 \mathrm{~m}, \mathrm{SNR}=30 \mathrm{~dB}, \mathrm{SIR}=0 \mathrm{~dB}$, FIR filter length is 15 , angle of desired source $\phi=90$ degrees, critical sampling for each case. Upper figure - frequency band 250-500 Hz, Lower figure - Frequency band 500-1000 Hz. 
Frequency Band 250-500 Hz

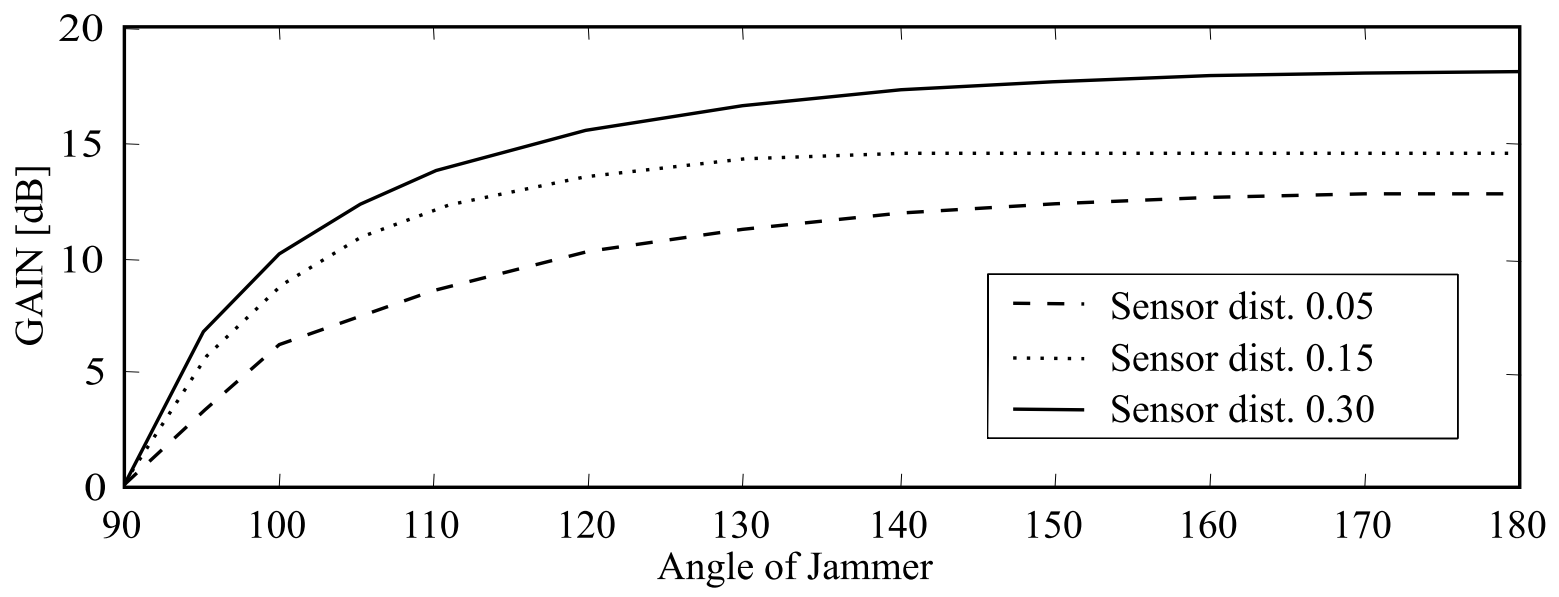

Frequency Band 500-1000 Hz

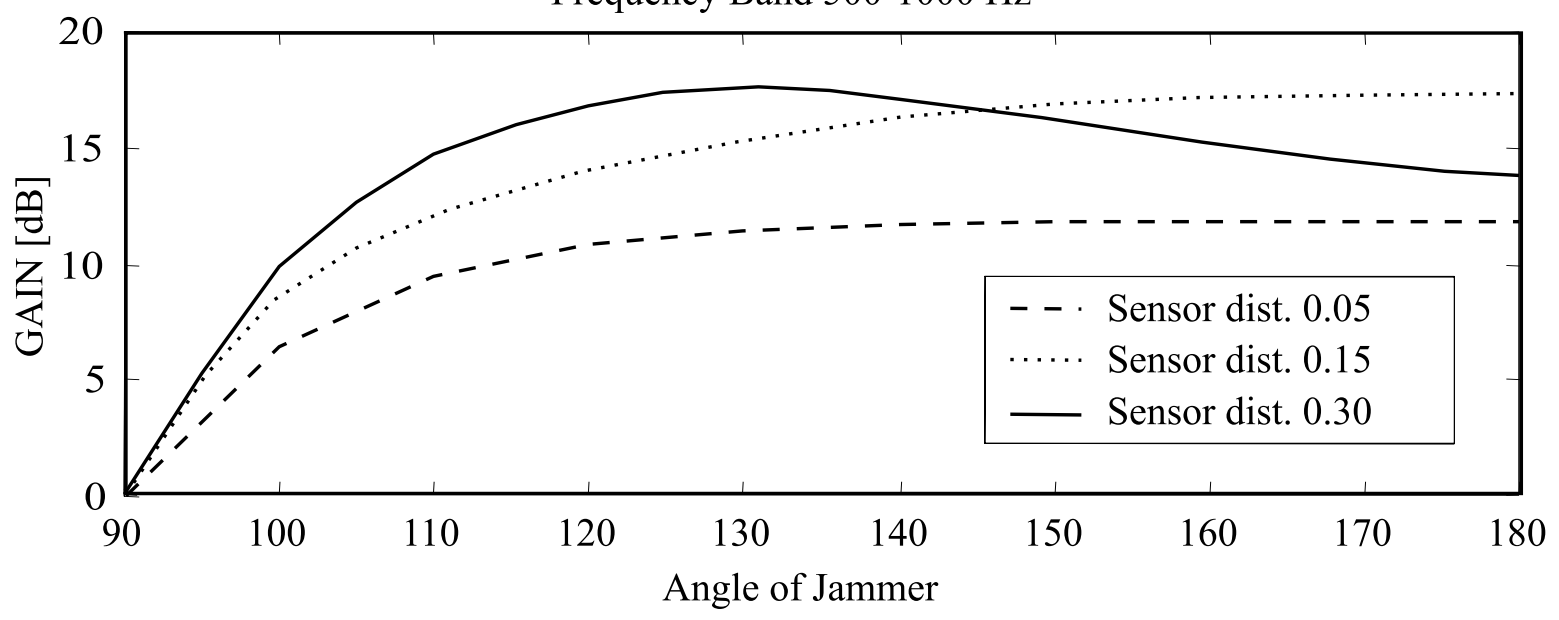

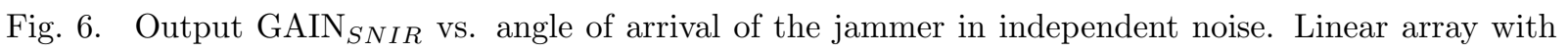
$\mathrm{N}=5$, sensor distance is chosen as $0.05,0.15$ and $0.30 \mathrm{~m}, \mathrm{SNR}=30 \mathrm{~dB}, \mathrm{SIR}=0 \mathrm{~dB}, \mathrm{FIR}$ filter length is 15 , angle of desired source $\phi=90$ degrees, critical sampling for each case. Upper figure - frequency band 250-500 Hz, Lower figure - Frequency band 500-1000 Hz. 


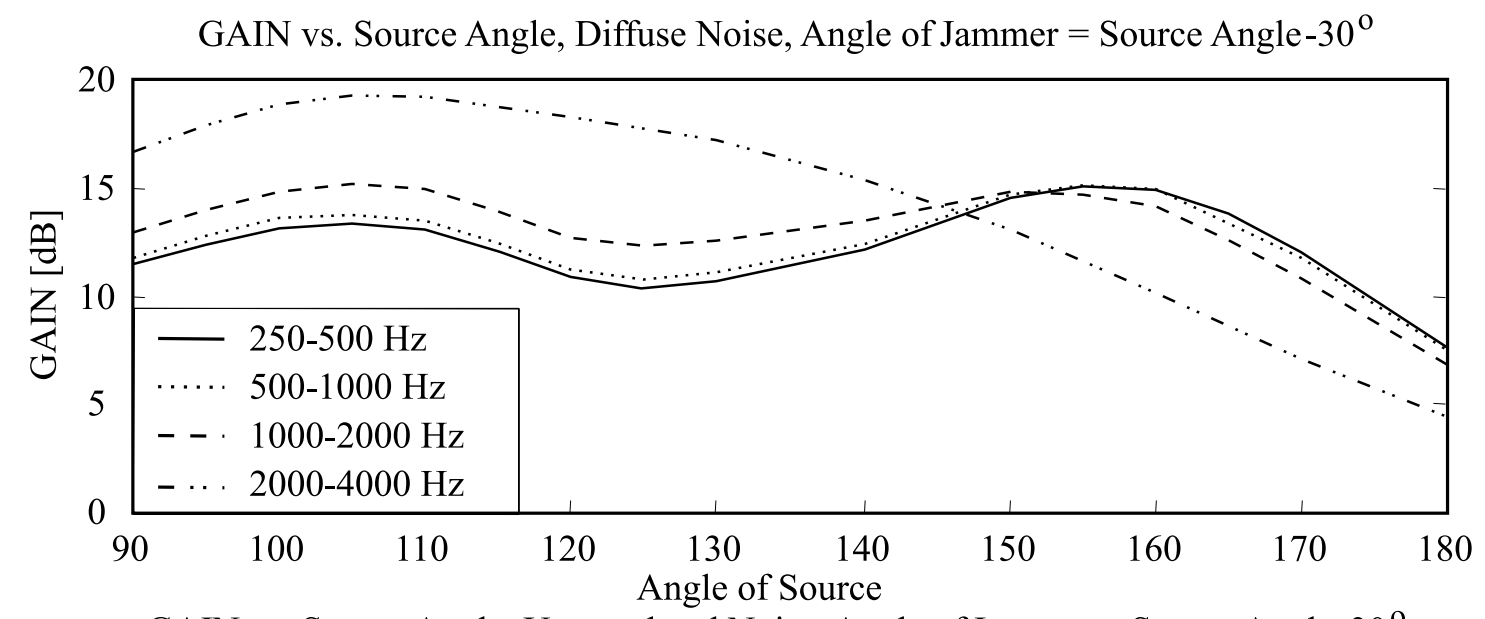

GAIN vs. Source Angle, Uncorrelated Noise, Angle of Jammer $=$ Source Angle $-30^{\circ}$

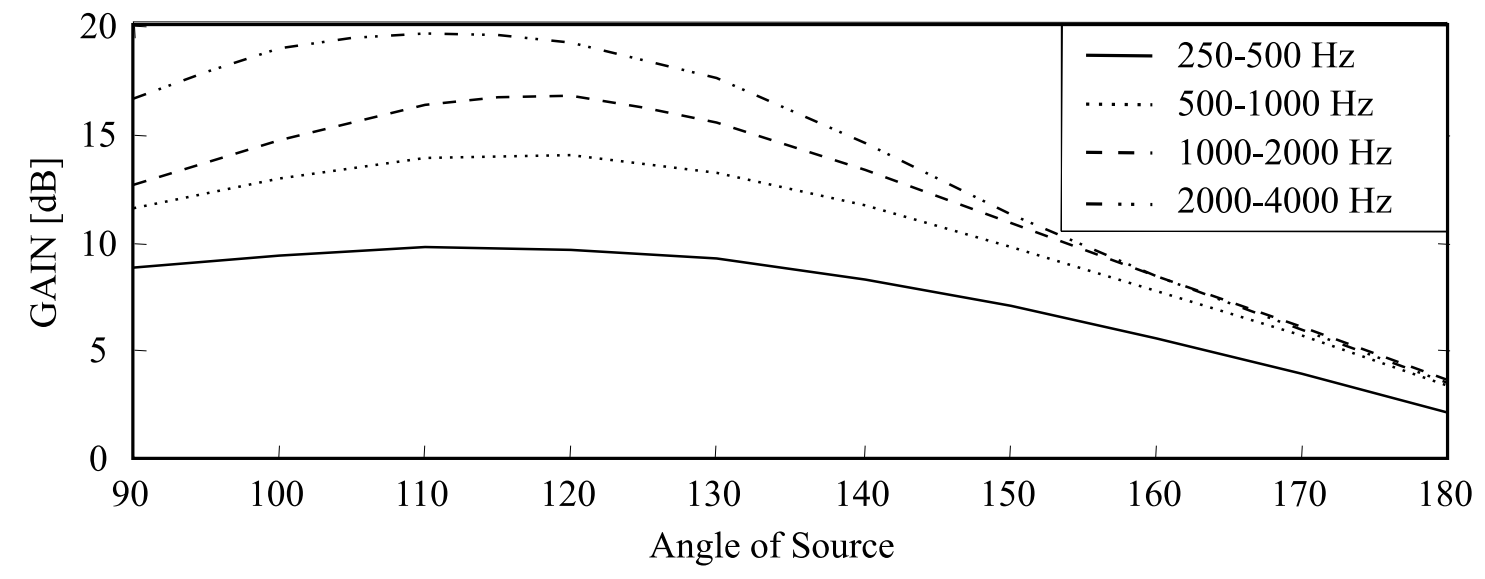

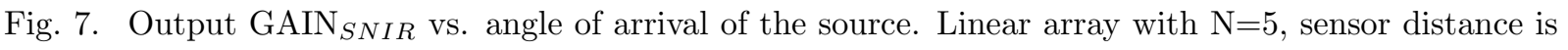
chosen as $0.05 \mathrm{~m}, \mathrm{SNR}=30 \mathrm{~dB}, \mathrm{SIR}=0 \mathrm{~dB}$, angle of jammer source $\theta=\phi-30$ degrees. Upper figure - diffuse noise field, Lower figure - independent noise. 


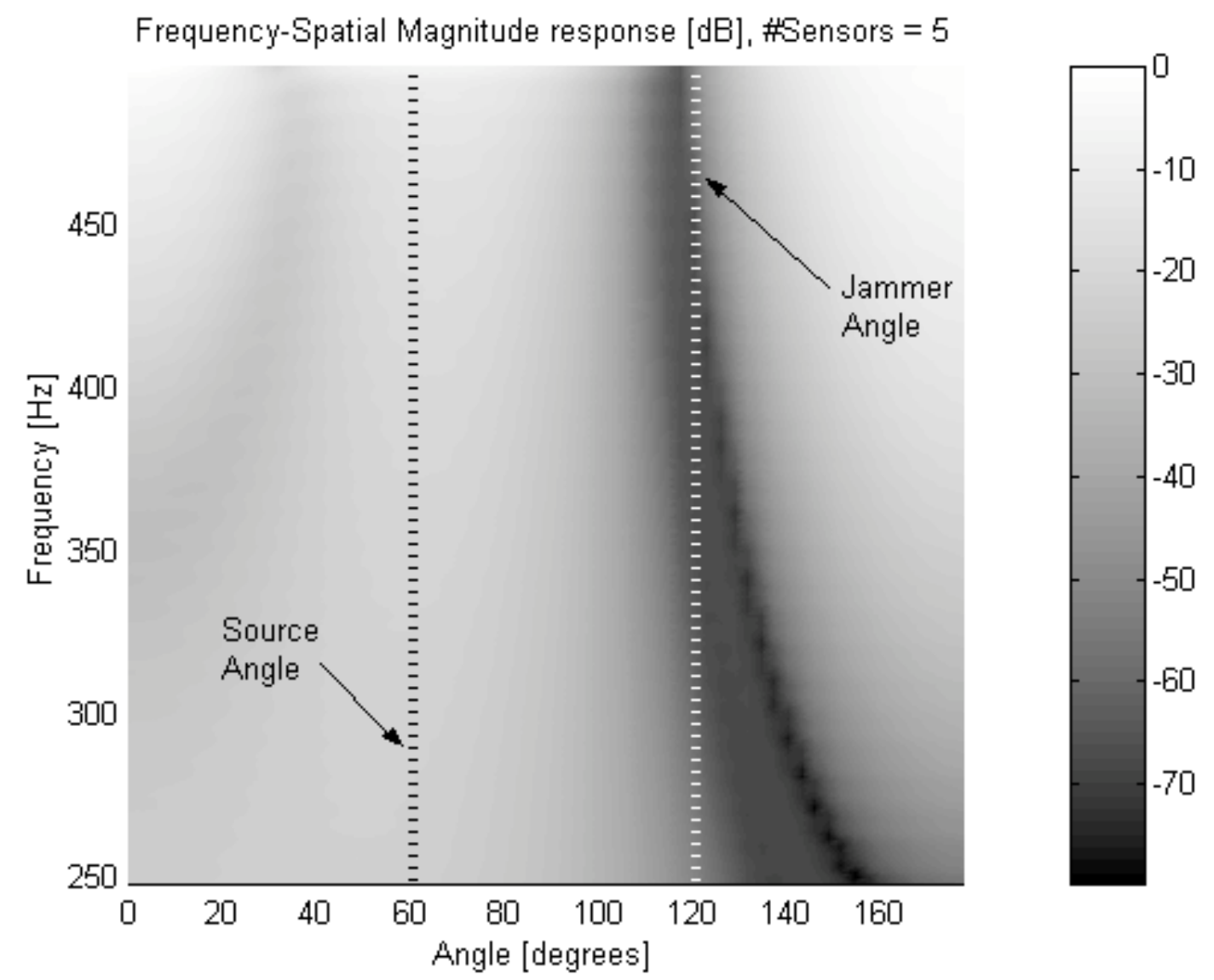

Fig. 8. Magnitude response of linear array with $\mathrm{N}=5$, sensor distance is $0.05 \mathrm{~m}, \mathrm{SNR}=30 \mathrm{~dB}, \mathrm{SIR}=0 \mathrm{~dB}$, FIR filter length is 15 , angle of desired source $\phi=60$ degrees and angle of jammer source $\theta=120$ degrees, $f_{s}=1 \mathrm{kHz}$ sampling frequency. Frequency range 250-500 Hz. 


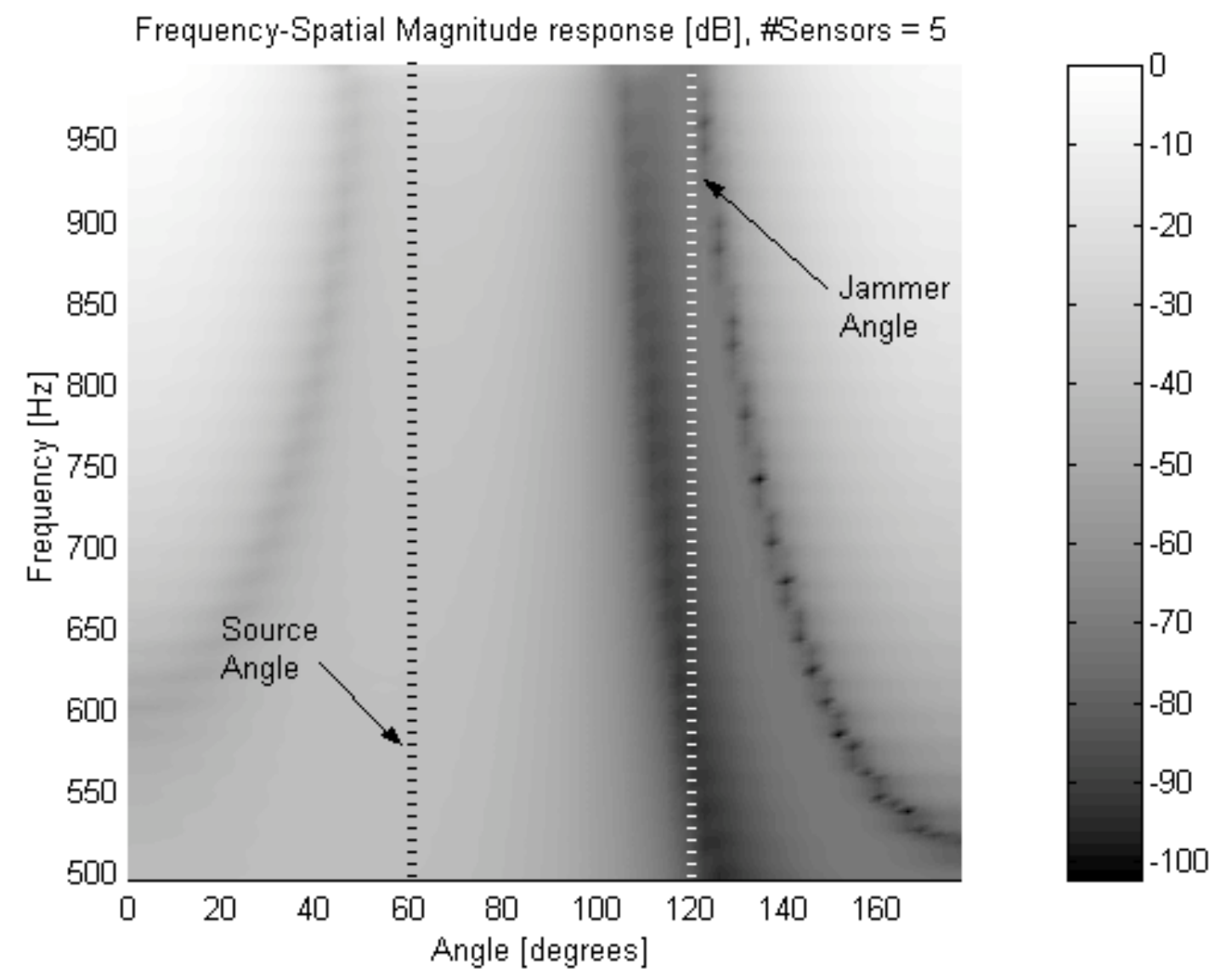

Fig. 9. Magnitude response of linear array with $\mathrm{N}=5$, sensor distance is $0.05 \mathrm{~m}, \mathrm{SNR}=30 \mathrm{~dB}, \mathrm{SIR}=0 \mathrm{~dB}$, FIR filter length is 15 , angle of desired source $\phi=60$ degrees and angle of jammer source $\theta=120$ degrees, $f_{s}=2 \mathrm{kHz}$ sampling frequency. Frequency range 500-1000 Hz. 